\title{
Uni- vs. Multiloculated Pelvic Lymphoceles: Differences in the Treatment of Symptomatic Pelvic Lymphoceles after Open Radical Retropubic Prostatectomy
}

\author{
Adrian Treiyer, Bjorn Haben, Eberhard Stark, Peter Breitling, Joachim Steffens \\ Department of Urology and Pediatric Urology, St. Antonius Hospital, Eschweiler, Germany
}

\begin{abstract}
Purpose: To evaluate the treatment of symptomatic pelvic lymphoceles (SPL) after performing radical retropubic prostatectomy (RRP) and pelvic lymphadenectomy (PLA) simultaneously.

Material and Methods: We analyzed, in a retrospective study, 250 patients who underwent RRP with PLA simultaneously. Only patients with SPL were treated using different non- and invasive procedures such as percutaneous aspiration, percutaneous catheter drainage (PCD) with or without sclerotherapy, laparoscopic lymphocelectomy (LL) and open marsupialization $(\mathrm{OM})$.

Results: Fifty-two patients (21\%) had postoperative subclinical pelvic lymphoceles. Thirty patients (12\%) developed SPL. Fifteen patients with noninfected uniloculated lymphocele (NUL) healed spontaneously after performing PCD. The remaining seven patients required sclerotherapy with additional doxycycline. After performing PCD, NUL healed better and faster than noninfected multiloculated lymphocele (NML) (success rate: $80 \%$ vs. 16\%, respectively). Twenty-seven percent of patients treated initially with PCD, with or without sclerotherapy had persistent lymphocele. All patients were successfully treated with LL. Only one patient had an abscess as a major complication of a persistent SPL after PCD and sclerotherapy and was treated via an open laparotomy.

Conclusions: Symptomatic NUL can be treated using PCD with or without sclerotherapy. If this therapy fails as first-line treatment, laparoscopic lymphocelectomy should be considered within a short period of time in order to achieve successful treatment. NML should be treated using a laparoscopic approach in centers where this type of expertise is available. Infected lymphoceles are drained externally. In these cases, percutaneous or open external drainage with adequate antibiotic coverage is preferable.
\end{abstract}

Key words: prostatic neoplasms; prostatectomy; pelvis; lymph nodes; lymphoceles; laparoscopy Int Braz J Urol. 2009; 35: 164-70

\section{INTRODUCTION}

A lymphocele, also known as a lymphocyst, is a collection of lymphatic fluid occurring as a consequence of surgical dissection and inadequate closure of afferent lymphatic vessels. In the literature, an incidence of $0.5-10 \%$ of patients treated by radical prostatectomy having symptomatic pelvic lymphoceles (SPL) postoperatively has been reported (1-3).

Pelvic lymphadenectomy (PLA) is frequently performed simultaneously with radical retropubic prostatectomy (RRP) to determine lymph node status (4). A surgical approach is indispensable since to date no imaging study can compare with PLA to detect the 
presence of metastasis $(5,6)$. However, this potential benefit must be weighed against the additional morbidity and costs associated with PLA.

To our knowledge there are only few up-todate studies focusing on the complications associated with PLA after RRP. Therefore, we were prompted to retrospectively analyze our data of postoperative SPL and the corresponding treatments to determine which procedure could be the most effective.

\section{MATERIALS AND METHODS}

Data on 250 patients who underwent RRP between January 2005 and December 2007 were collected. Patients were followed-up for a minimum of 6 months.

A limited or standard PLA was routinely performed after an open RRP. Our standard pelvic lymphadenectomy involved the dissection and removal of lymphatic tissue from the level of the external iliac vein to the obturator nerve, extending proximal to the common iliac artery bifurcation and distal to the proximal femoral canal to include the node of Cloquet. We did not perform an extended pelvic lymphadenectomy, which removes the lymphatic tissue surrounding the internal iliac vein and presacral region. After completing the surgery 2 closed suction drains were placed, each one laterally to the bladder, in relationship with the area of pelvic lymph node dissection. All patients received perioperative antibiotics and low molecular weight heparin after RRP.

In order to diagnose pelvic lymphoceles we routinely performed pelvic ultrasound after RRP and PLA. Pelvic ultrasound studies were performed as standard procedure during the first 10 days after RRP at least three times in each patient. In patients in whom pelvic lymphoceles were found, we performed daily ultrasound controls to check the progression or resolution of the fluid collections. Pelvic lymphoceles were defined as a pelvic fluid collection of more than $50 \mathrm{~mL}$ after drainage removal. Persistent lymphorrhea (PL) was diagnosed when catheter outputs exceeded $50 \mathrm{~mL}$ per day after 3 days of surgery. In these cases, we performed microbiological analyses of the pelvic fluid collections. Fluid collections with creatinine levels similar to serum were treated as lymphoceles. Cystograms were performed to distinguish between an anastomotic leak and a lymphocele. Doppler lower extremity studies were performed in all patients with signs and/or symptoms of complicated lymphoceles compressing the iliac veins. In major complicated pelvic lymphoceles with or without infections, we performed a CT scan or MRI.

The symptoms of this collection depended on the size and presence of infection. Patients with SPL may present a visible or palpable pelvic mass. Symptoms or signs may be a result of venous compression resulting in unilateral leg edema, leg pain and deep vein thrombosis. Fever and chills should suggest secondary infected pelvic lymphoceles.

PL and SPL were evaluated by controlling the fluid drainage per day $(\leq 50 \mathrm{~mL} /$ day or $\geq 50 \mathrm{~mL} /$ day $)$ or the size after drainage removal $(\leq 50 \mathrm{~mL}$. or $\geq 50$ $\mathrm{mL}$ ), respectively.

Treatment options also depended on other factors such as position, loculations and the recurrence of the collections. Noninfected uniloculated lymphoceles (NUL) were primarily treated using percutaneous catheter drainage (PCD) with or without additionally sclerotherapy. Noninfected multiloculated lymphoceles (NML) and persistent lymphoceles after PCD with or without sclerotherapy were treated using laparoscopic lymphocelectomy (LL).

SPL were treated initially with PCD. Percutaneous drainage was performed after insertion of an 8 to $14 \mathrm{~F}$ pigtail catheter using ultrasound guidance. The catheter was sutured in place and daily output was recorded. Resolution of fluid collection was determined by follow-up ultrasound and clinical symptoms.

PL was treated initially with additional sclerotherapy for a maximum of 10 consecutive days. Sclerotherapy was performed with doxycycline (40 $\mathrm{mg} /$ day) instillated through the drainage (drain after RRP or drain after percutaneous drainage) using an aseptic technique. Lymphocele recurrence after one course of sclerotherapy was not managed with a second attempt using these sclerosant agents. If this therapy failed, we occluded the drainage for 24 hours to control, with ultrasound, the size of the lymphatic collection. We removed the catheter when the collection remained equal and did not increase. In these cases with the growing size of the lymphatic cavity, 
as well as recurrence of lymphocele or with PL after PCD and sclerotherapy we performed a LL.

Laparoscopic lymphocelectomy was performed as described by $\mathrm{McCullough}$ et al. using a 3 or 4-port technique depending on whether the approach was uni or bilateral (7).

Open laparotomy was only performed in rare cases with persistent lymphocele after percutaneous and/or laparoscopic approaches failed, and also in major complications of the pelvic lymphoceles such as infections, abscess or acute bleeding after using other techniques.

\section{RESULTS}

Three experienced surgeons performed 250 RRPs with limited PLA. The median number of lymph nodes removed was 12.5 (r: 1-42).

Fifty-two patients (overall rate: $21 \%$ ) had subclinical pelvic lymphoceles after RRP (Ultrasound volume range: $50-300 \mathrm{~mL}$ ). Forty patients developed unilateral lymphoceles and only 12 bilateral. Thirty patients (23 unilateral/7 bilateral) (overall rate: 12\%) developed SPL. In 15 cases after PCD, there was spontaneous resolution of the symptoms and they were treated using routine ultrasound surveillance. The remaining fifteen patients had PL and were treated with PCD and sclerotherapy in 7 cases. Another 3 patients were treated successfully using LL after a combined PCD-sclerotherapy failed. In other 4 cases LL was performed after PCD without sclerotherapy failed. In only one patient we performed an open laparatomy because of an infected complicated lymphocele (Table-1).

Patients with NUL who underwent PCD and sclerotherapy as first-line-treatment had a higher success rate compared to those with a NML ( $80 \%$ vs. $16 \%$, respectively) (Table-2).

Twenty-seven percent of patients who were initially treated with PCD with or without sclerotherapy had a PL. All of them (100\%) were successfully treated with laparoscopic marsupialization and intraoperative drainage removal.

We also observed that those patients treated successfully with PCD and adjuvant sclerotherapy required additional days of treatment to eliminate the persistent lymphorrhea compared to those initially treated with LL (average of 9.5 days of treatment vs. 1 day, respectively).

In a small group of patients (n: 4) after performing PCD we did not instill sclerosing agents in the lymphatic cavity. In these cases we decided to directly perform LL due to a persistent lymphorrhea. In all these patients we achieved good results with no recurrences of lymphoceles after this approach.

Table 1 - Pelvic lymphoceles after pelvic lymphadenectomy and RRP.

Initially asymptomatic pelvic lymphoceles (Uni/Bilateral) $1 \mathrm{n} / 2 \mathrm{n}$ (overall (\%))

$40 / 12(20.8)$

Persistent / progressed symptomatic pelvic lymphoceles (Uni/Bilateral) 1n/2n (overall (\%))

Spontaneously regressed pelvic lymphoceles with $\mathrm{PCD}$ alone

Persistent symptomatic pelvic lymphoceles after PCD

PCD with sclerotherapy

Laparoscopic marsupialization of pelvic lymphoceles after a failed combined PCD-sclerotherapy

Laparoscopic marsupialization of pelvic lymphoceles without using sclerotherapy

Open laparotomy

$R R P=$ radical retropubic prostatectomy; $P C D=$ percutaneous catheter drainage. 
Table 2 - Classification of symptomatic pelvic lymphoceles and the results after performing percutaneous catheter drainage (PCD).

\begin{tabular}{lcc}
\hline Patients with Symptomatic Pelvic Lymphoceles & N = 30 & $\begin{array}{c}\text { N of Persistent Lymphoceles after } \\
\text { Percutaneous Catheter Drainage (\%) }\end{array}$ \\
\hline Noninfected uniloculated lymphoceles & 25 & $4(16.0)$ \\
Noninfected multiloculated lymphoceles & 5 & $4(80.0)$ \\
Infected lymphocele & $1^{*}$ & $1(100.0)$ \\
\hline
\end{tabular}

* After performing a PCD in a noninfected uniloculated lymphocele, one patient developed an infected lymphocele.

Open laparotomy was performed because of an abscess as a major complication of a symptomatic secondary infected lymphocele. After removal of the infection the patient had no further complications.

As major complication there were 2 patients (overall rate: $0.8 \%$ ) who developed a deep venous thrombosis and leg edema. The presence of pulmonary emboli was not observed either radiographically or scintigraphically.

\section{COMMENTS}

In our data a high incidence $(21 \%)$ of subclinical lymphoceles after PLA and RRP was observed. However, our rate was lower than that originally obtained when any sonographically or radiographically detected lymphocele was considered (range: 27-61\%) $(8,9)$. Despite an incidence of $21 \%$, in the current study the overall rate of clinically significant SPL after PLA and RRP was $12 \%$. This observation is in agreement with the results described by other series $(3,10-12)$. Pepper (3), Solberg (8) and Campbell (10) reported symptomatic or clinically significant lymphoceles in $3.5 \%, 2.3 \%$ and $1.6 \%$ of patients, respectively.

Another relevant consequence of lymphoceles is the significantly higher incidence of re-intervention. In our study approximately $50 \%$ of all re-interventions performed in patients with prostatectomy were related to lymphocele management. In a recent study by Musch et al. these authors described similar results (4).

Symptomatic lymphoceles can be managed initially by PCD with or without instillation of sclerosing agents, such as tetracycline, ampicillin, ethanol, doxycycline or povidone-iodine $(1,3)$. If the lymphocele is nonloculated, sclerosant therapy may be attempted (13). A multiloculated lymphocyst as shown in our study has more chances to recur under sclerotherapy because of the multiple cysts in the lymphocele cavity.

However, lymphocele recurrence rates are high: 50 to $100 \%$ (14) after simple aspiration and 10 to $15 \%$ (15) following sclerosant therapy. In our data we found lymphocele recurrence in $27 \%$ of patients treated initially with PCD with or without sclerotherapy. In our experience percutaneous sclerotherapy is associated with a low success rate and possible contamination of the lymphocele cavity. In the best case scenario Teruel et al. (15) described successful sclerotherapy using long-term percutaneous catheter drainage and at least two daily instillations of the sclerosant agent for an average of 25 days (up to a maximum of 45 days). Contrary to this concept we performed a short-term sclerotherapy for no more than 10 consecutive days. It may be possible that this once daily short-term therapy was the cause of a higher lymphocele recurrence in our data compared to other studies.

However, the long-term treatment of PCD to achieve higher success rates, prompted us to use more frequently the laparoscopic marsupialization of lymphocele, which was successful in all patients. In the literature more than $90 \%$ success was reported after peritoneal marsupialization $(3,16)$. Pelvic lymphoceles appear to be suited ideally for drainage by laparoscopic techniques. The bulging wall of the lymphocele cavity is usually readily apparent laparoscopically. We did not routinely perform omentoplasty during laparoscopic lymphocelectomy. Disadvantages of this technique include the requirement for a gen- 
eral anesthetic, and surgical trauma compared to a percutaneous approach. However, we consider that a decreased analgesic requirement, shorter hospitalization and a more rapid recovery are advantages to more frequently perform laparoscopy and therefore this approach should be considered as the standard therapy for a noninfected symptomatic lymphocele when the percutaneous sclerotherapy fails as first line-treatment. We suggest that when SPL persists, having previously attempted a noninvasive procedure, then after a short period of time a laparoscopic intraperitoneal drainage approach should be performed to avoid a secondary infection of the lymphocele cavity or an unsuccessfully extended time of noninvasive therapy.

Post-laparoscopy recurrence warrants open surgical marsupialization with or without omentoplasty (13).

Symptomatic infected lymphoceles require meticulous imaging surveillance (Ultrasound or CT scan control) and more invasive therapy is needed if major complications such as septicemia, fever $\geq$ $39.5^{\circ} \mathrm{C}$, progression of an infected lymphocele or abscess occur. In some cases PCD can be attempted. As regards these complications some studies remain controversial. There are studies reporting a high recurrence rate after performing percutaneous drainage, whereas other authors report good results. We believe that a percutaneous approach should be performed in patients who are stable and have a localized controlled infected lymphocele. If this approach fails an open technique should be performed.

Although we performed a limited PLA instead of an extensive technique on all patients in our study, we obtained a significantly high median number of pelvic lymph nodes (median No. 12.5 lymph nodes per PLA). According to other studies the risk of lymphocele is significantly higher as the number of removed lymph nodes increases (1). This could possibly explain our higher incidence of pelvic lymphoceles compared with other data.

We suspect that in some patients the use of 2 closed suction drains instead of drainage without suction may have increased the incidence of pelvic lymphoceles reported in our study. However, further studies should be performed in order to confirm this suspicion.
Another promoter of lymphoceles in our study population might have been the standardized perioperative administration of low dose heparin for thromboembolism prophylaxis, in accordance with German Association of the Scientific Medical Societies Guidelines. Bigg and Catalona (17), and Tomic et al. (18) identified low dose heparin as a factor causing increased lymph secretion and a higher rate of lymphocele formation. In our patients heparin was administered exclusively subcutaneously into the upper arm to avoid increased lymph secretion in the pelvis (19).

\section{CONCLUSIONS}

Simple percutaneous aspiration should be used only for diagnostic purposes when indicated.

In our experience percutaneous catheter drainage with sclerotherapy is associated with a low success rate, need for a long period of treatment to achieve success and possible contamination of the lymphocele cavity. However, PCD with sclerotherapy could be attempted in patients with nonloculated symptomatic lymphoceles as first line treatment.

Our data suggest that laparoscopic lymphocelectomy appears to be safe and effective, with minimal postoperative morbidity and a low recurrence rate. Therefore, if percutaneous catheter drainage with or without sclerotherapy fails as first-line treatment, laparoscopy marsupialization of pelvic lymphocele should be considered within a short period of time. In some specific cases, as in multiloculated lymphoceles, laparoscopic lymphocelectomy should be considered as first-line treatment at centers where this type of expertise is available.

When infected lymphoceles are drained externally, percutaneous or open external drainage with adequate antibiotic coverage should be performed.

\section{REFERENCES}

1. Hsu TH, Gill IS, Grune MT, Andersen R, Eckhoff D, Goldfarb DA, et al.: Laparoscopic lymphocelectomy: a multi-institutional analysis. J Urol. 2000; 163: 1096-8; discussion 1098-9. 
2. Augustin H, Hammerer P, Graefen M, Palisaar J, Noldus J, Fernandez S, et al.: Intraoperative and perioperative morbidity of contemporary radical retropubic prostatectomy in a consecutive series of 1243 patients: results of a single center between 1999 and 2002. Eur Urol. 2003; 43: 113-8.

3. Pepper RJ, Pati J, Kaisary AV: The incidence and treatment of lymphoceles after radical retropubic prostatectomy. BJU Int. 2005; 95: 772-5.

4. Musch M, Klevecka V, Roggenbuck U, Kroepfl D: Complications of pelvic lymphadenectomy in 1,380 patients undergoing radical retropubic prostatectomy between 1993 and 2006. J Urol. 2008; 179: 923-8; discussion 928-9.

5. Joseph JV, Greene T: Re: Standard versus limited pelvic lymph node dissection for prostate cancer in patients with a predicted probability of nodal metastasis greater than $1 \% \mathrm{~K}$. Touijer, F. Rabbani, J. R. Otero, F. P. Secin, J. A. Eastham, P. T. Scardino And B. Guillonneau J Urol 2007; 178: 120-124. J Urol. 2008; 179: 789-90.

6. Borley N, Fabrin K, Sriprasad S, Mondaini N, Thompson $\mathrm{P}$, Muir G, et al.: Laparoscopic pelvic lymph node dissection allows significantly more accurate staging in "high-risk" prostate cancer compared to MRI or CT. Scand J Urol Nephrol. 2003; 37: 382-6.

7. McCullough CS, Soper NJ, Clayman RV, So SS, Jendrisak MD, Hanto DW: Laparoscopic drainage of a posttransplant lymphocele. Transplantation. 1991; 51: 725-7.

8. Solberg A, Angelsen A, Bergan U, Haugen OA, Viset T, Klepp O: Frequency of lymphoceles after open and laparoscopic pelvic lymph node dissection in patients with prostate cancer. Scand J Urol Nephrol. 2003; 37: 218-21.

9. Spring DB, Schroeder D, Babu S, Agee R, Gooding GA: Ultrasonic evaluation of lymphocele formation after staging lymphadenectomy for prostatic carcinoma. Radiology. 1981; 141: 479-83.
10. Campbell SC, Klein EA, Levin HS, Piedmonte MR: Open pelvic lymph node dissection for prostate cancer: a reassessment. Urology. 1995; 46: 352-5.

11. Briganti A, Chun FK, Salonia A, Suardi N, Gallina A, Da Pozzo LF, et al.: Complications and other surgical outcomes associated with extended pelvic lymphadenectomy in men with localized prostate cancer. Eur Urol. 2006; 50: 1006-13.

12. Heidenreich A, Varga Z, Von Knobloch R: Extended pelvic lymphadenectomy in patients undergoing radical prostatectomy: high incidence of lymph node metastasis. J Urol. 2002; 167: 1681-6.

13. Gill IS, Hodge EE, Munch LC, Goldfarb DA, Novick AC, Lucas BA: Transperitoneal marsupialization of lymphoceles: a comparison of laparoscopic and open techniques. J Urol. 1995; 153: 706-11.

14. Kay R, Fuchs E, Barry JM: Management of postoperative pelvic lymphoceles. Urology. 1980; 15: 345-7.

15. Teruel JL, Escobar EM, Quereda C, Mayayo T, Ortuño J: A simple and safe method for management of lymphocele after renal transplantation. J Urol. 1983; 130: 1058-9.

16. Meyers AM, Levine E, Myburgh JA, Goudie E: Diagnosis and management of lymphoceles after renal transplantation. Urology. 1977; 10: 497-502.

17. Bigg SW, Catalona WJ: Prophylactic mini-dose heparin in patients undergoing radical retropubic prostatectomy. A prospective trial. Urology. 1992; 39: 309-13.

18. Tomic R, Granfors T, Sjödin JG, Ohberg L: Lymph leakage after staging pelvic lymphadenectomy for prostatic carcinoma with and without heparin prophylaxis. Scand J Urol Nephrol. 1994; 28: 273-5.

19. Kröpfl D, Krause R, Hartung R, Pfeiffer R, Behrendt $\mathrm{H}$ : Subcutaneous heparin injection in the upper arm as a method of avoiding lymphoceles after lymphadenectomies in the lower part of the body. Urol Int. 1987; 42: 416-23.

\section{Correspondence address:}

Dr. Adrián Treiyer

St. Antonius Hospital

Department of Urology

Dechant-Deckers Str. 8

Eschweiler, 52249, Germany

E-mail: aetreiyer@yahoo.com.ar 


\section{EDITORIAL COMMENT}

The article is an excellent clinical paper and should be read by all clinicians who perform pelvic lymphadenectomies because it demonstrates the good clinical practice considering the handling of pelvic lymphoceles. We share similar experience with laparoscopic treatment of lymphoceles and prefer this treatment because of his almost universal and immediate efficiency.

Dr. Darko Kröpfl Department of Urology Kliniken Essen Mitte Essen, Germany E-mail:d.kroepf@kliniken-essen-mitte.de

\section{EDITORIAL COMMENT}

The authors deserve praise for this very interesting retrospective study about the occurrence of lymphoceles after radical prostatectomy with associated pelvic lymphadenectomy. The occurrence of $21 \%$ (52 patients) of lymphoceles detected by abdominal ultrasound, of which $12 \%$ (30 patients) with symptomatic lymphoceles, is superior to the average reported in the literature in recent years (1), which is probably a reflection of a stricter definition adopted by the authors instead of a greater occurrence in comparison to what was obtained by other authors.

The use of laparoscopic drainage was relatively low - 7 cases -, all with good evolution, which is coherent with the previously published experience concerning the laparoscopic treatment of lymphoceles resulting from renal transplant (2).

The authors suggest that drainage without suction (with Penrose drain) could be better than tubular drains. This is the subjective impression of some surgeons, but this has to be proved.

Some authors have published good results without drainage after open or robotic radical prostatectomy $(3,4)$. They argue that the routine placement of a pelvic drain may not be required. This is an interesting issue to be investigated.

\section{REFERENCES}

1. Pepper RJ, Pati J, Kaisary AV: The incidence and treatment of lymphoceles after radical retropubic prostatectomy. BJU Int. 2005; 95: 772-5.

2. Castilho LN, Ferreira U, Liang LS, Fregonesi A, Netto Jr NR. Lymphocele post renal transplantation: videolaparoscopic treatment: report of five cases and review of the literature. Braz J Urol. 1997; 23: 17-22.

3. Araki M, Manoharan M, Vyas S, Nieder AM, Soloway MS: A pelvic drain can often be avoided after radical retropubic prostatectomy--an update in 552 cases. Eur Urol. 2006; 50: 1241-7; discussion 1246-7.

4. Sharma S, Kim HL, Mohler JL: Routine pelvic drainage not required after open or robotic radical prostatectomy. Urology. 2007; 69: 330-3.

Dr. Lisias N. Castilho Catholic University of Campinas Campinas, SP, Brazil E-mail:lisias@dglnet.com.br 